International Journal of Pure and Applied Mathematics

Volume 88 No. 4 2013, 537-546

ISSN: 1311-8080 (printed version); ISSN: 1314-3395 (on-line version)

url: http://www.ijpam.eu

doi: http://dx.doi.org/10.12732/ijpam.v88i4.8

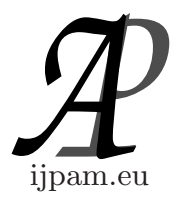

\title{
A PARAMETER FOR RAMANUJAN'S FUNCTION $\chi(q)$ OF DEGREE 9 AND THEIR EXPLICIT EVALUATION
}

B.N. Dharmendra ${ }^{1}$, M.R. Rajesh Kanna ${ }^{2}$, S. Vasanth Kumar ${ }^{3}$

Post Graduate Department of Mathematics

Maharani's Science College for Women

J.L.B. Road, Mysore, 570 001, INDIA

${ }^{3}$ Research and Development Center

Bharathiar University

Coimbathore, 641 046, INDIA

Abstract: In this paper, we study a ratio's of parameter for Ramanujan's function $\chi(q)$ of degree 9 and their explicit values.

AMS Subject Classification: 33D10, 40A15, 11A55, 30B70

Key Words: continued fraction, theta functions

\section{Introduction}

In Chapter 16 of his second notebook [1], Ramanujan develops the theory of theta-function and is defined by

$$
\begin{aligned}
f(a, b) & :=\sum_{n=-\infty}^{\infty} a^{\frac{\mathrm{n}(\mathrm{n}+1)}{2}} b^{\frac{\mathrm{n}(\mathrm{n}-1)}{2}}, \quad|a b|<1, \\
& =(-a ; a b)_{\infty}(-b ; a b)_{\infty}(a b ; a b)_{\infty}
\end{aligned}
$$

where $(a ; q)_{0}=1$ and $(a ; q)_{\infty}=(1-a)(1-a q)\left(1-a q^{2}\right) \cdots$.

Received: August 29, 2013

(C) 2013 Academic Publications, Ltd.

$\S_{\text {Correspondence author }}$ url: www.acadpubl.eu 
Following Ramanujan, we defined

$$
\begin{gathered}
\varphi(q):=f(q, q)=\sum_{n=-\infty}^{\infty} q^{n^{2}}=\frac{(-q ;-q)_{\infty}}{(q ;-q)_{\infty}}, \\
\psi(q):=f\left(q, q^{3}\right)=\sum_{n=0}^{\infty} q^{\frac{\mathrm{n}(\mathrm{n}+1)}{2}}=\frac{\left(q^{2} ; q^{2}\right)_{\infty}}{\left(q ; q^{2}\right)_{\infty}}, \\
f(-q):=f\left(-q,-q^{2}\right)=\sum_{n=-\infty}^{\infty}(-1)^{n} q^{\frac{\mathrm{n}(3 \mathrm{n}-1)}{2}}=(q ; q)_{\infty}
\end{gathered}
$$

and

$$
\chi(q):=\left(-q ; q^{2}\right)_{\infty} .
$$

Now we define a modular equation in brief. The ordinary hypergeometric series

${ }_{2} F_{1}(a, b ; c ; x)$ is defined by

$$
{ }_{2} F_{1}(a, b ; c ; x):=\sum_{n=0}^{\infty} \frac{(a)_{n}(b)_{n}}{(c)_{n} n !} x^{n},
$$

where $(a)_{0}=1,(a)_{n}=a(a+1)(a+2) \cdots(a+n-1)$ for any positive integer $n$, and $|x|<1$.

Let

$$
z:=z(x):={ }_{2} F_{1}\left(\frac{1}{2}, \frac{1}{2} ; 1 ; x\right)
$$

and

$$
q:=q(x):=\exp \left(-\pi \frac{{ }_{2} F_{1}\left(\frac{1}{2}, \frac{1}{2} ; 1 ; 1-x\right)}{{ }_{2} F_{1}\left(\frac{1}{2}, \frac{1}{2} ; 1 ; x\right)}\right),
$$

where $0<x<1$.

Let $r$ denote a fixed natural number and assume that the following relation holds:

$$
r \frac{{ }_{2} F_{1}\left(\frac{1}{2}, \frac{1}{2} ; 1 ; 1-\alpha\right)}{{ }_{2} F_{1}\left(\frac{1}{2}, \frac{1}{2} ; 1 ; \alpha\right)}=\frac{{ }_{2} F_{1}\left(\frac{1}{2}, \frac{1}{2} ; 1 ; 1-\beta\right)}{{ }_{2} F_{1}\left(\frac{1}{2}, \frac{1}{2} ; 1 ; \beta\right)} .
$$

Then a modular equation of degree $r$ in the classical theory is a relation between $\alpha$ and $\beta$ induced by (8). We often say that $\beta$ is of degree $r$ over $\alpha$ and $m:=\frac{z(\alpha)}{z(\beta)}$ is called the multiplier. We also use the notations $z_{1}:=z(\alpha)$ and $z_{r}:=z(\beta)$ to indicate that $\beta$ has degree $r$ over $\alpha$. 
The function $\chi(q)$ is intimately connected to Ramanujan's class invariants $G_{n}$ an $g_{n}$ which are defined by

$$
G_{n}=2^{-1 / 4} q^{-1 / 24} \chi(q), \quad g_{n}=2^{-1 / 4} q^{-1 / 24} \chi(-q)
$$

where $q=e^{-\pi^{\vee} \bar{n}}$ and $n$ is a positive rational number. Since from [1, Entry $12(\mathrm{v}),(\mathrm{vi}), \mathrm{p} .56]$

$$
\begin{gathered}
\chi(q)=2^{1 / 6}\left\{\alpha(1-\alpha) q^{-1}\right\}^{-1 / 24} \\
\chi(-q)=2^{1 / 6}(1-\alpha)^{1 / 12} \alpha^{-1 / 24} q^{-1 / 24}
\end{gathered}
$$

Nipen Saikia [4] introduce the parameter $I_{m, n}$ which is defined as

$$
I_{m, n}:=\frac{\chi(q)}{q^{(-m+1) / 24} \chi\left(q^{m}\right)}, \quad q=e^{-\pi \sqrt{n / m}},
$$

where $m$ and $n$ are positive real numbers.

In Section 3, we study the modular relation between $I_{9, n}$ and $I_{9, k^{2} n}$, their explicit evaluations of $I_{9, n}$ for $n=2,3,5$ and 7 .

\section{Preliminary Results}

Lemma 1. [3] If $P=\frac{\varphi(q) \varphi\left(q^{2}\right)}{\varphi\left(q^{9}\right) \varphi\left(q^{18}\right)}$ and $Q=\frac{\varphi(q) \varphi\left(q^{18}\right)}{\varphi\left(q^{9}\right) \varphi\left(q^{2}\right)}$, then

$$
\begin{aligned}
& Q^{2}+\frac{1}{Q^{2}}+\left(P^{2}+\frac{9^{2}}{P^{2}}\right)+2\left(P+\frac{9}{P}\right)\left[4+3\left(Q+\frac{1}{Q}\right)\right]=8\left(Q+\frac{1}{Q}\right) \\
& +4\left[\left(\sqrt{P^{3}}+\frac{3^{3}}{\sqrt{P^{3}}}\right)\left(\sqrt{Q}+\frac{1}{\sqrt{Q}}\right)-\left(\sqrt{P}+\frac{3}{\sqrt{P}}\right)\left(\sqrt{Q^{3}}+\frac{1}{\sqrt{Q^{3}}}\right)+3\right] .
\end{aligned}
$$

Lemma 2. [3] If $P=\frac{\varphi(q)}{\varphi\left(q^{9}\right)}$ and $Q=\frac{\varphi\left(q^{3}\right)}{\varphi\left(q^{27}\right)}$, then

$$
\left(3-P-\frac{3}{P}\right)\left(3-Q-\frac{3}{Q}\right)=\left(\frac{Q}{P}\right)^{2} \text {. }
$$


Lemma 3. [3] If $P=\frac{\varphi(q) \varphi\left(q^{5}\right)}{\varphi\left(q^{9}\right) \varphi\left(q^{45}\right)}$ and $Q=\frac{\varphi(q) \varphi\left(q^{45}\right)}{\varphi\left(q^{9}\right) \varphi\left(q^{5}\right)}$, then

$$
\begin{aligned}
& Q^{3}+\frac{1}{Q^{3}}-15\left(Q^{2}+\frac{1}{Q^{2}}\right)-45\left(Q+\frac{1}{Q}\right)-\left(P^{2}+\frac{81}{P^{2}}\right)-10\left(P+\frac{9}{P}\right) \\
& \times\left[2+Q+\frac{1}{Q}\right]+5\left(\sqrt{P^{3}}+\frac{3^{3}}{\sqrt{P^{3}}}\right)\left(\sqrt{Q}+\frac{1}{\sqrt{Q}}\right)+15\left(\sqrt{P}+\frac{3}{\sqrt{P}}\right) \\
& \times\left[\left(\sqrt{Q^{3}}+\frac{1}{\sqrt{Q^{3}}}\right)+2\left(\sqrt{Q}+\frac{1}{\sqrt{Q}}\right)\right]=40 .
\end{aligned}
$$

Lemma 4. [3] If $P=\frac{\varphi(q) \varphi\left(q^{7}\right)}{\varphi\left(q^{9}\right) \varphi\left(q^{63}\right)}$ and $Q=\frac{\varphi(q) \varphi\left(q^{63}\right)}{\varphi\left(q^{9}\right) \varphi\left(q^{7}\right)}$, then

$$
\begin{aligned}
& Q^{4}+\frac{1}{Q^{4}}-35\left(Q^{3}+\frac{1}{Q^{3}}\right)-413\left(Q^{2}+\frac{1}{Q^{2}}\right)-1379\left(Q+\frac{1}{Q}\right)-1694 \\
& -\left(P^{3}+\frac{9^{3}}{P^{3}}\right)-7\left(P^{2}+\frac{9^{2}}{P^{2}}\right)\left[7+3\left(Q+\frac{1}{Q}\right)\right]-21\left(P+\frac{9}{P}\right) \\
& {\left[21+14\left(Q+\frac{1}{Q}\right)+3\left(Q^{2}+\frac{1}{Q^{2}}\right)\right]+7\left(\sqrt{P^{5}}+\frac{3^{5}}{\sqrt{P^{5}}}\right)\left(\sqrt{Q}+\frac{1}{\sqrt{Q}}\right)} \\
& +63\left[\sqrt{P}+\frac{3}{\sqrt{P}}\right]\left[7\left(\sqrt{Q^{3}}+\frac{1}{\sqrt{Q^{3}}}\right)+14\left(\sqrt{Q}+\frac{1}{\sqrt{Q}}\right)+\left(\sqrt{Q^{5}}+\frac{1}{\sqrt{Q^{5}}}\right)\right] \\
& +21\left(\sqrt{P^{3}}+\frac{3^{3}}{\sqrt{P^{3}}}\right)\left[2\left(\sqrt{Q^{3}}+\frac{1}{\sqrt{Q^{3}}}\right)+7\left(\sqrt{Q}+\frac{1}{\sqrt{Q}}\right)\right]=0 .
\end{aligned}
$$

Lemma 5. [5, p.56]

$$
\frac{f^{3}(q)}{q f^{3}\left(q^{9}\right)}=\frac{\varphi^{2}(q)}{\varphi^{2}\left(q^{9}\right)}\left\{\frac{\varphi(q)-3 \varphi\left(q^{9}\right)}{\varphi\left(q^{9}\right)-\varphi(q)}\right\} .
$$

Lemma 6. [1, Ch. 16, Entry 24(iii), p.39]

$$
\chi(q)=\frac{\varphi(q)}{f(q)} .
$$

Lemma 7. [4]

$$
I_{m, 1}=1
$$

Lemma 8. [4]

$$
I_{m, n} I_{m, 1 / n}=1
$$




\section{General Theorems and Explicit Evaluations of $I_{m, n}$}

$$
\begin{gathered}
\text { Theorem 9. If } P:=q \frac{\chi(q) \chi\left(q^{2}\right)}{\chi\left(q^{9}\right) \chi\left(q^{18}\right)} \text { and } Q:=q^{-1 / 3} \frac{\chi(q) \chi\left(q^{18}\right)}{\chi\left(q^{9}\right) \chi\left(q^{2}\right)} \text { then } \\
\left\{P^{3}+\frac{1}{P^{3}}\right\}+7\left\{P^{2}+\frac{1}{P^{2}}\right\}+14\left\{P+\frac{1}{P}\right\}-\left\{Q^{3}+\frac{1}{Q^{3}}\right\}+16 \\
=\left\{\sqrt{Q^{3}}+\frac{1}{\sqrt{Q^{3}}}\right\}\left[\left\{\sqrt{P^{5}}+\frac{1}{\sqrt{P^{5}}}\right\}+4\left\{\sqrt{P^{3}}+\frac{1}{\sqrt{P^{3}}}\right\}+5\left\{\sqrt{P}+\frac{1}{\sqrt{P}}\right\}\right]
\end{gathered}
$$

Proof. Replace $q$ by $q^{9}$ in the lemma (6), we obtain

$$
\chi\left(q^{9}\right)=\frac{\varphi\left(q^{9}\right)}{f\left(q^{9}\right)} .
$$

Dividing the equations (18) by (22), we get

$$
\frac{\chi(q)}{\chi\left(q^{9}\right)}=\frac{\varphi(q)}{\varphi\left(q^{9}\right)} \frac{f\left(q^{9}\right)}{f(q)} .
$$

Raising the power three and also multiplying $q$ on both side of the above equation (23), we get

$$
q \frac{\chi^{3}(q)}{\chi^{3}\left(q^{9}\right)}=\frac{\varphi^{3}(q)}{\varphi^{3}\left(q^{9}\right)}\left\{q \frac{f^{3}\left(q^{9}\right)}{f^{3}(q)}\right\}
$$

Using the equation (17) and the above equation (24), we obtain

$$
P^{3}+P(a-1)-3 a=0
$$

where $P:=\frac{\varphi(q)}{\varphi\left(q^{9}\right)}, a:=q \frac{\chi^{3}(q)}{\chi^{3}\left(q^{9}\right)}$, solve the above equation we get, where $b=P^{2}$, solve the above equation we get

$$
b=\frac{1-a+\sqrt{a^{2}+10 a+1}}{2}
$$

using the above equation (26) and equation (13), we obtain 


$$
\begin{aligned}
& \left(y^{3}+x^{3}+4 y^{4} x^{7}-16 y^{4} x^{4}+4 y^{4} x+y^{5} x^{8}-14 y^{5} x^{5}+5 y^{5} x^{2}+y x^{7}+4 y x^{4}\right. \\
& +5 y^{2} x^{5}+4 y^{7} x^{4}+y^{7} x-y^{7} x^{7}-7 y^{2} x^{2}+5 x^{6} y^{3}-7 x^{6} y^{6}-14 x^{3} y^{3}+5 x^{3} y^{6} \\
& \left.+y^{8} x^{5}-y x\right)\left(8 y^{5} x^{14}+y^{16} x^{10}+y^{14} x^{2}+y^{14} x^{14}+9 y^{14} x^{8}-y^{14} x^{11}-9 y^{8} x^{5}\right. \\
& +8 y^{14} x^{5}+5 y^{13} x^{10}-5 y^{13} x^{13}-3 y^{13} x^{7}-5 y^{13} x^{4}+6 y^{4} x^{7}+27 y^{4} x^{4}+y^{4} x \\
& +6 y^{7} x^{10}+y^{6}-3 y^{7} x^{13}-9 y^{5} x^{8}+y^{10} x^{16}-26 y^{5} x^{11}-44 y^{5} x^{5}-y^{5} x^{2}-y^{11} x^{14} \\
& +15 y^{4} x^{10}-5 y^{4} x^{13}+9 y^{8} x^{14}-4 y x^{7}+y x^{4}+6 y^{10} x^{7}+15 y^{10} x^{4}-y^{10} x \\
& +5 y^{10} x^{13}+50 y^{10} x^{10}+27 y^{8} x^{8}-9 y^{8} x^{11}+8 y^{2} x^{11}-y^{2} x^{5}-y x^{10}+6 y^{7} x^{4} \\
& -4 y^{7} x-33 y^{7} x^{7}+y^{2} x^{2}+y^{2} x^{14}+9 y^{2} x^{8}-3 x^{9} y^{3}-33 x^{9} y^{9}+6 x^{9} y^{12}+9 y^{8} x^{2} \\
& -5 x^{12} y^{3}+15 x^{12} y^{6}+6 x^{12} y^{9}+27 x^{12} y^{12}+6 x^{9} y^{6}-4 x^{15} y^{9}+x^{12} y^{15}-9 y^{11} x^{8} \\
& +5 x^{6} y^{3}+50 x^{6} y^{6}+6 x^{6} y^{9}+15 x^{6} y^{12}-5 x^{3} y^{3}+5 x^{3} y^{6}-3 x^{3} y^{9}-5 x^{3} y^{12} \\
& \left.-x^{6} y^{15}-4 x^{9} y^{15}-x^{15} y^{6}+x^{15} y^{12}+x^{6}-44 y^{11} x^{11}-26 y^{11} x^{5}+8 y^{11} x^{2}\right)=0
\end{aligned}
$$

where $x=q^{1 / 3} \frac{\chi(q)}{\chi\left(q^{9}\right)}$ and $y=q^{2 / 3} \frac{\chi\left(q^{2}\right)}{\chi\left(q^{18}\right)}$, by examining the behavior of the above factors near $q=0$, we can find a neighborhood about the origin, where the first factor is zero; whereas other factor are not zero in this neighborhood. By the Identity Theorem first factor vanishes identically. This completes the proof.

Remark 1. Here by using the definition of (12), then above theorem (9) is also can be written as $P=I_{9, n} I_{9,4 n}$ and $Q=\frac{I_{9, n}}{I_{9,4 n}}$

Corollary 10. We have

$$
\begin{aligned}
& I_{9,2}=\left[\frac{(\sqrt{3}+\sqrt{2})(3 \sqrt{3}-5)}{\sqrt{2}}\right]^{1 / 3}, \\
& I_{9,1 / 2}=\left[\frac{(\sqrt{3}-\sqrt{2})(3 \sqrt{3}+5)}{\sqrt{2}}\right]^{1 / 3}, \\
& I_{9,4}=\left[\frac{\sqrt{6}(1+\sqrt{3})-2-2 \sqrt{(3+\sqrt{3})(\sqrt{3}-\sqrt{2})}}{4}\right],
\end{aligned}
$$




$$
I_{9,1 / 4}=\left[\frac{\sqrt{6}(1+\sqrt{3})-2+2 \sqrt{(3+\sqrt{3})(\sqrt{3}-\sqrt{2})}}{4}\right]
$$

Proof. Setting $n=1 / 2$ in Theorem (9) and using the Lemma (8), we obtain

$$
A^{12}+20 A^{9}-60 A^{6}+20 A^{3}+1=0
$$

where $A:=I_{9, n}<1$, by solving the above equation, we obtain (28) and (29).

Again setting $n=1$ in Theorem (9) and using Lemma (7), we get

$$
B^{8}+4 B^{7}-2 B^{6}-8 B^{5}-8 B^{4}-8 B^{3}-2 B^{2}+4 B+1=0
$$

where $B:=I_{9,4 n}<1$, the above equation can be written as

$$
D^{4}+4 D^{3}-6 D^{2}-20 D-2=0
$$

where $D:=B+\frac{1}{B}$, solving the above equation (34), we obtain (30) and (31).

Theorem 11. If $P:=q^{4 / 3} \frac{\chi(q) \chi\left(q^{3}\right)}{\chi\left(q^{9}\right) \chi\left(q^{27}\right)}$ and $Q:=q^{-2 / 3} \frac{\chi(q) \chi\left(q^{27}\right)}{\chi\left(q^{9}\right) \chi\left(q^{3}\right)}$ then

$$
\begin{aligned}
& 74+\left\{P^{6}+\frac{1}{P^{6}}\right\}+35\left\{Q^{3}+\frac{1}{Q^{3}}\right\}+8\left\{Q^{6}+\frac{1}{Q^{6}}\right\}-\left\{Q^{9}+\frac{1}{Q^{9}}\right\} \\
& +7\left\{P^{3}+\frac{1}{P^{3}}\right\}\left[28+19\left\{Q^{3}+\frac{1}{Q^{3}}\right\}\right]+\left\{Q^{6}+\frac{1}{Q^{6}}\right\}\left[28+19\left\{P^{3}+\frac{1}{P^{3}}\right\}\right] \\
& +10\left\{\sqrt{P^{9}}+\frac{1}{\sqrt{P^{9}}}\right\}\left\{\sqrt{Q^{3}}+\frac{1}{\sqrt{Q^{3}}}\right\}=10\left\{\sqrt{P^{3}}+\frac{1}{\sqrt{P^{3}}}\right\}\left[\left\{\sqrt{Q^{15}}+\frac{1}{\sqrt{Q^{15}}}\right\}\right. \\
& \left.-17\left\{\sqrt{Q^{9}}+\frac{1}{\sqrt{Q^{9}}}\right\}-46\left\{\sqrt{Q^{3}}+\frac{1}{\sqrt{Q^{3}}}\right\}\right] .
\end{aligned}
$$


Proof. Employing the equations (26) and equation (14), we obtain

$$
\begin{gathered}
10 x^{6} y^{3}-x^{18} y^{6}+35 x^{12} y^{6}+19 y^{15} x^{9}+8 y^{15} x^{3}+17 x^{12} y^{3}+10 x^{15} y^{12} \\
+10 x^{12} y^{15}-y^{18} x^{3}+x^{3} y^{3}+x^{15} y^{15}-x^{18} y^{3}-x^{6} y^{18}+19 x^{3} y^{9}-x^{15} \\
-y^{12}-x^{18}-y^{18}+17 y^{15} x^{6}+17 y^{12} x^{3}+28 x^{12} y^{12}+28 x^{6} y^{6}+10 x^{3} y^{6} \\
+74 x^{9} y^{9}+35 x^{6} y^{12}+46 x^{9} y^{12}+46 x^{6} y^{9}+19 x^{9} y^{3}+19 x^{15} y^{9}+8 x^{15} y^{3} \\
+46 x^{12} y^{9}+17 x^{15} y^{6}-x^{12}-y^{15}+46 x^{9} y^{6}=0
\end{gathered}
$$

where $x=q^{1 / 3} \frac{\chi(q)}{\chi\left(q^{9}\right)}$ and $y=q \frac{\chi\left(q^{3}\right)}{\chi\left(q^{27}\right)}$. This completes the proof.

Remark 2. Here by using the definition of (12), then above theorem (11) is also can be written as $P=I_{9, n} I_{9,9 n}$ and $Q=\frac{I_{9, n}}{I_{9,9 n}}$

Corollary 12. We have

$$
\begin{aligned}
& I_{9,3}=\left[2^{1 / 3}-1\right]^{1 / 3}, \\
& I_{9,1 / 3}=\left[2^{2 / 3}+2^{1 / 3}+1\right]^{1 / 3}, \\
& I_{9,9}=\left[\frac{u^{2}+3 u+13-\sqrt{u^{4}+34 u^{2}+78 u+451+12 \sqrt{3}}}{u}\right]^{1 / 3}, \\
& I_{9,1 / 9}=\left[\frac{u^{2}+3 u+13+\sqrt{u^{4}+34 u^{2}+78 u+451+12 \sqrt{3}}}{u}\right]^{1 / 3} .
\end{aligned}
$$

where $u:=(47+2 \sqrt{3})^{1 / 3}$

Proof. Setting $n=1 / 3$ in Theorem (4) and using the Lemma (8), we obtain

$$
\begin{array}{r}
\left(A^{9}+3 A^{6}+3 A^{3}-1\right)\left(A^{9}-3 A^{6}-3 A^{3}-1\right)(A+1)^{2}\left(A^{2}-A+1\right)^{2}\left(A^{2}+1\right)^{2} \\
\left(A^{4}-A^{2}+1\right)^{2}=0, \quad(41)
\end{array}
$$

where $A:=I_{9, n}<1$, by solving the first factor we obtain (37) and similarly by solving the second factor we get (38), the other factors does not satisfying the condition of $I_{9,9}<1$.

Again setting $n=1$ in Theorem (11) and using Lemma (7), we obtain 


$$
54 B^{15}+135 B^{12}-3 B^{18}+135 B^{6}+54 B^{3}+204 B-3=0
$$

where $B:=I_{9,9 n}<1$, the above equation can be written as

$$
3 M^{3}-54 M^{2}-144 M-96=0
$$

where $M:=B^{3}+\frac{1}{B^{3}}$, solving the above equation (43), we get (39) and (40)

Theorem 13. If $P:=q^{2} \frac{\chi(q) \chi\left(q^{5}\right)}{\chi\left(q^{9}\right) \chi\left(q^{45}\right)}$ and $Q:=q^{-4 / 3} \frac{\chi(q) \chi\left(q^{45}\right)}{\chi\left(q^{9}\right) \chi\left(q^{5}\right)}$ then

$$
10+5\left\{P+\frac{1}{P}\right\}+\left\{Q^{3}+\frac{1}{Q^{3}}\right\}=5\left\{\sqrt{P}+\frac{1}{\sqrt{P}}\right\}\left\{\sqrt{Q^{3}}+\frac{1}{\sqrt{Q^{3}}}\right\} .
$$

Proof. Employing the equation (26) and equation (15), we obtain (44)

Remark 3. Here by using the definition of (12), then above theorem (13) is also can be written as $P=I_{9, n} I_{9,25 n}$ and $Q=\frac{I_{9, n}}{I_{9,25 n}}$

Corollary 14. We have

$$
\begin{gathered}
I_{9,5}=[4-\sqrt{15}]^{1 / 3}, \\
I_{9,1 / 5}=[4+\sqrt{15}]^{1 / 3} . \\
I_{9,25}=\frac{2+\sqrt{15}-\sqrt{4 \sqrt{15}+15}}{2} . \\
I_{5,1 / 25}=\frac{2+\sqrt{15}+\sqrt{4 \sqrt{15}+15}}{2} .
\end{gathered}
$$

Proof. Employing Theorem (13), Lemma (8) and (7), we arrive (45)-(48)

Theorem 15. If $P:=q^{8 / 3} \frac{\chi(q) \chi\left(q^{7}\right)}{\chi\left(q^{9}\right) \chi\left(q^{63}\right)}$ and $Q:=q^{-2} \frac{\chi(q) \chi\left(q^{63}\right)}{\chi\left(q^{9}\right) \chi\left(q^{7}\right)}$ then

$$
\begin{aligned}
& \left\{Q^{4}+\frac{1}{Q^{4}}\right\}-14\left\{Q^{2}+\frac{1}{Q^{2}}\right\}-35\left\{Q+\frac{1}{Q}\right\}-\left\{P^{3}+\frac{1}{P^{3}}\right\} \\
& 7\left\{\sqrt{Q^{3}}+\frac{1}{\sqrt{Q^{3}}}\right\}\left[\left\{\sqrt{P^{3}}+\frac{1}{\sqrt{P^{3}}}\right\}+3\left\{\sqrt{P}+\frac{1}{\sqrt{P}}\right\}\right] .
\end{aligned}
$$


Proof. Employing the equations (26) and equation (16), we obtain (49).

Remark 4. Here by using the definition of (12), then above theorem (13) is also can be written as $P=I_{9, n} I_{9,49 n}$ and $Q=\frac{I_{9, n}}{I_{9,49 n}}$

Corollary 16. We have

$$
\begin{gathered}
I_{9,7}=\frac{1+\sqrt{21}-\sqrt{6+2 \sqrt{21}}}{4} . \\
I_{9,1 / 7}=\frac{1+\sqrt{21}+\sqrt{6+2 \sqrt{21}}}{4} \\
I_{9,49}=\frac{1}{4}[4+3 \sqrt{7}+\sqrt{X}-\sqrt{2 X+8 \sqrt{X}+6 \sqrt{7 X}}] . \\
I_{9,1 / 49}=\frac{1}{4}[4+3 \sqrt{7}+\sqrt{X}+\sqrt{2 X+8 \sqrt{X}+6 \sqrt{7 X}}] .
\end{gathered}
$$

where $X=63+24 \sqrt{7}$.

Proof. Employing Theorem (15), Lemma (8) and (7), we arrive (50)-(53)

\section{References}

[1] B. C. Berndt, Ramanujan's Notebooks, Part III, Springer-Verlag, New York (1991).

[2] M. S. Mahadeva Naika, Some theroems on Ramanujan cubic continued fraction and related ientities. Tamsui Oxf. J. Math. Sci. 24(3) (2008), 243256.

[3] M. S. Mahadeva Naika, K. Susan Bairy and S. Chandankumar, On some explicit evaluation of the ratios of Ramanujan's theta-function. (Communicated).

[4] Nipen Saikia, Modular Idendities and Explicit Values of a New Continued Fraction of Ramanujan, Global Journal of Mathematical Sciences, Volume 4, Number 3, pp. 245-248.India (2012).

[5] S. Ramanujan, The 'lost' notebook and other unpublished papers. New Delhi. Narosa (1988). 